\title{
Photon echo transients from an inhomogeneous ensemble of semiconductor quantum dots
}

\author{
S. V. Poltavtsev,${ }^{1,2, *}$ M. Salewski, ${ }^{1}$ Yu. V. Kapitonov ${ }^{3}$ I. A. Yugova,${ }^{2}$ I. A. Akimov,${ }^{1,4}$ \\ C. Schneider, ${ }^{5}$ M. Kamp, ${ }^{5}$ S. Höfling, ${ }^{5}$ D. R. Yakovlev, ${ }^{1,4}$ A. V. Kavokin, ${ }^{2,6,7}$ and M. Bayer ${ }^{1,4}$ \\ ${ }^{1}$ Experimentelle Physik 2, Technische Universität Dortmund, 44221 Dortmund, Germany \\ ${ }^{2}$ Spin Optics Laboratory, St. Petersburg State University, 198504 St. Petersburg, Russia \\ ${ }^{3}$ St. Petersburg State University, 199034 St. Petersburg, Russia \\ ${ }^{4}$ Ioffe Physical-Technical Institute, Russian Academy of Sciences, 194021 St. Petersburg, Russia \\ ${ }^{5}$ Technische Physik, Universität Würzburg, 97074 Würzburg, Germany \\ ${ }^{6}$ School of Physics and Astronomy, University of Southampton, SO17 1 BJ, Southampton, United Kingdom \\ ${ }^{7}$ CNR-SPIN, Viale del Politecnico 1, I-00133 Rome, Italy
}

(Dated: February 4, 2016)

\begin{abstract}
An ensemble of quantum dot excitons may be used for coherent information manipulation. Due to the ensemble inhomogeneity any optical information retrieval occurs in form of a photon echo. We show that the inhomogeneity can lead to a significant deviation from the conventional echo timing sequence. Variation of the area of the initial rotation pulse, which generates excitons in a dot subensemble only, reveals this complex picture of photon echo formation. We observe a retarded echo for $\pi / 2$ pulses, while for $3 \pi / 2$ the echo is advanced in time as evidenced through monitoring the Rabi oscillations in the time-resolved photon echo amplitude from (In,Ga)As/GaAs self-assembled quantum dot structures and confirmed by detailed calculations.

PACS numbers: 42.50.Ex, 42.50.Md, 42.25.Kb, 42.70.Nq
\end{abstract}

Manipulation of electronic states by laser pulses requires precise timing of the pulse sequence. A particular example is ultrafast coherent control of exciton complexes confined in semiconductor nanostructures ${ }^{1}$ which may be used for manipulating quantum states ${ }^{2-4}$ or even storing quantum information ${ }^{5,6}$. For an inhomogeneous ensemble of two-level systems (TLS) such as quantum dots (QDs) the information retrieval induces echoes for the recovered signal ${ }^{7-9}$. To be of practical use, the echo formation time needs to be determined with high accuracy.

The scenario is well established when a weakly inhomogeneous ensemble of TLS is resonantly addressed by ultrashort laser pulses whose spectrum covers the distribution of optical transitions in the ensemble. In this conventional picture of a photon echo (PE), a first excitation pulse creates a macroscopic polarization which subsequently dephases due to inhomogeneity. A second subsequent optical rephasing pulse retrieves the macroscopic polarization ${ }^{10}$ with subsequent emission of a $\mathrm{PE}$ pulse delayed by a time exactly equal to the interval between the rephasing and excitation pulse, $\tau_{12}$. Typically the temporal profile of the PE pulse has a Gaussian shape reflecting the inhomogeneous frequency distribution of the excited ensemble.

In semiconductors this picture may be complicated by several features: (1) Due to the many-body interactions a description by a distribution of independent TLS is as a rule not appropriate, but complex coherent transients are recorded ${ }^{11,12}$. However, localization of excitons strongly reduces exciton-exciton interactions ${ }^{13}$. Therefore, QDs with particularly strong three-dimensional localization can be often reasonably well considered as TLS with long coherence times. Indeed, Rabi oscillations of exciton complexes were demonstrated in QDs using various techniques ${ }^{14-17}$. (2) In addition, fluctuations in QD size, shape and composition lead to a considerable inhomogeneous broadening of the optical transitions in QDs. Simultaneously excitation with ultrashort pulses having a broader spectrum than the inhomogeneity in many cases is prohibited because of the demand of addressing a specific optical transition. The relatively narrow energy spacing of different electronic excitations limits the spectral width that the laser pulses may have, to avoid excitation of multiple transitions.

Therefore a careful consideration of the optical frequency detuning with respect to the TLS optical resonance is mandatory when considering the PE transient formation, even in the limit of small excitation levels. Generally, it is expected that for strongly inhomogeneous TLS ensembles the PE temporal profile may undergo significant distortion compared to the standard $\tau_{12}-2 \tau_{12}$ sequence, showing either retarded or advanced echo pulses ${ }^{18}$. This is due to the non-negligible inhomogeneity-induced dephasing of oscillators during the optical excitation pulse, so that the finite pulse duration should be taken into account. Surprisingly, these variations of the PE transients in TLS were only reported in ruby ${ }^{19}$, where the characteristic excitation and depolarization times are in the range of tens of nanoseconds, i.e. orders of magnitude longer than in semiconductor nanostructures. So far, the PE transients in QDs were considered in the conventional $\tau_{12}-2 \tau_{12}$ picture only.

In this paper, we demonstrate that the $\mathrm{PE}$ transients are strongly influenced by the inhomogeneity of a QD ensemble. We study the dynamics of the PE from an ensemble of (In,Ga)As/GaAs semiconductor QDs excited by picosecond laser pulses. By varying the amplitude of 

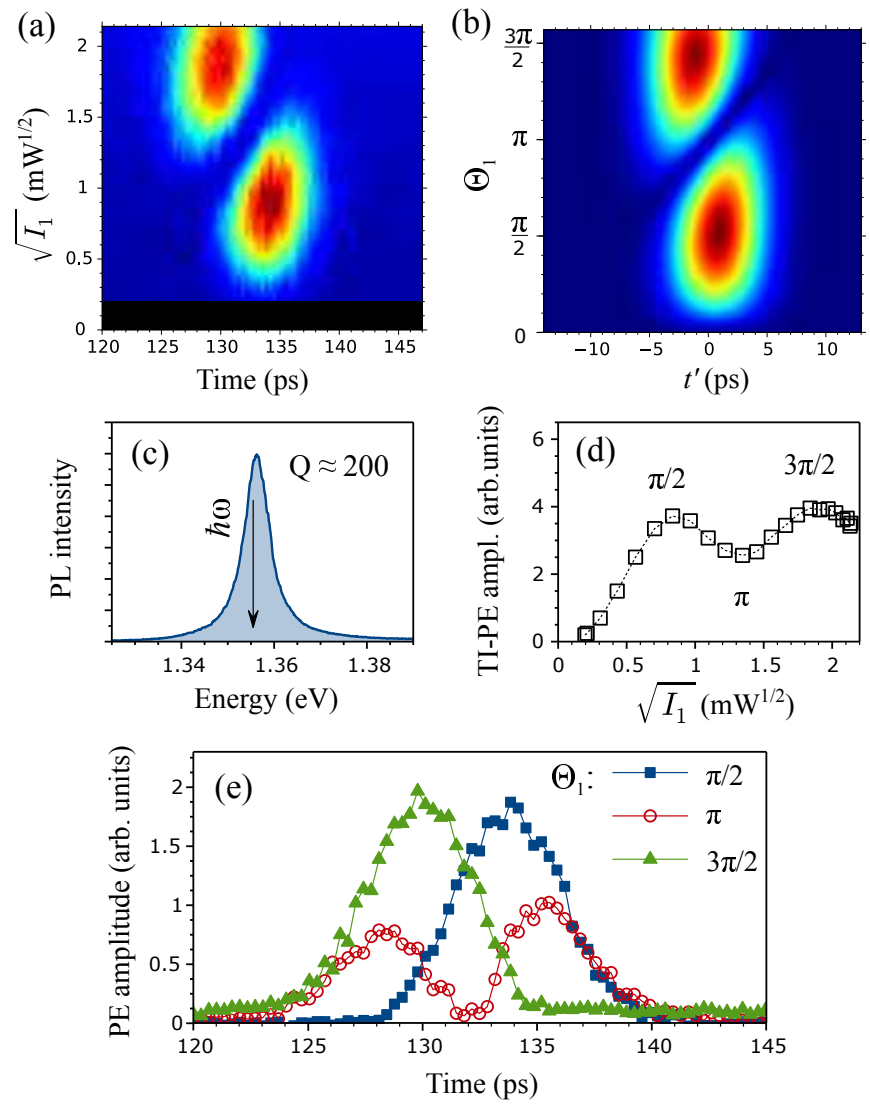

FIG. 1: (Color online) Experimental data and theoretical calculations of the absolute value of PE amplitude. (a) Experimental dependence of the PE temporal profile on the first pulse amplitude $\sqrt{I_{1}}$. (b) Theoretical modeling of the data from (a) using Eq. (2) with the following parameters: pump duration $\tau_{p}=2.9 \mathrm{ps}$, inhomogeneity $2 \sqrt{2 \ln 2} \hbar \delta \approx 0.65 \mathrm{meV}$, and rephasing pulse area $\Omega_{2} \tau_{p}=\pi$. In the colormaps, darkblue indicates the zero level, dark-red corresponds to maximum signal. (c) PL spectrum of the sample with the energy position of excitation, $\hbar \omega$, indicated by the arrow. (d) Timeintegrated (TI) modulus of the PE amplitude measured as function of the first pulse amplitude. (e) PE transients for $\Theta_{1}=\pi / 2, \pi$ and $3 \pi / 2$, respectively.

the first excitation pulse we find a complex evolution of Rabi oscillations in the amplitude of the PE, deviating strongly from the $\tau_{21}-2 \tau_{21}$ expectation. Namely, we observe temporal shifts of the $\mathrm{PE}$ pulse relative to the expected PE arrival time. We analyze the experimental results measured on excitons using a transparent analytical model.

The PE experiment was carried out on a sample with a single layer of (In, Ga)As QDs which is inserted into a GaAs $\lambda$-microcavity. The QD density is about $1.8 \times 10^{9}$ $\mathrm{cm}^{-2}$ and the barrier contains a Si $\delta$-layer with donor density $8 \times 10^{9} \mathrm{~cm}^{-2}$, separated from the QD-layer by 10 $\mathrm{nm}$. The Bragg mirrors consist of alternating GaAs and AlAs layers with thicknesses of $68 \mathrm{~nm}$ and $82 \mathrm{~nm}$, respectively. They have 5 and 18 such pairs in the top and bottom mirror, respectively. With a microcavity $Q$-factor of $\approx 200$, the photon lifetime is much shorter than the laser pulse duration of several picoseconds. Therefore, the main influence of the microcavity is to enhance the lightmatter interaction for those QDs with optical frequency in resonance with the cavity mode. Further, the broad exciton photoluminescence (PL) spectrum from the QDs with a width of $30 \mathrm{meV}$ is convoluted with the relatively narrow photon cavity mode to result in a relatively narrow emission band with full-width of $6.5 \mathrm{meV}$. The energy of the cavity mode depends on the sample spot due to the wedged cavity design. A PL spectrum measured at the studied sample spot discussed subsequently is shown in Fig. 1(c).

To study the PE dynamics, a degenerate transient four-wave-mixing (FWM) experiment was performed in reflection geometry. The sample was placed into a helium bath cryostat and cooled down to a temperature of $\sim 2 \mathrm{~K}$. We used a tunable mode-locked Ti:Sa laser as source of the optical pulses with duration of about $2.5 \mathrm{ps}$ and repetition rate of about $76 \mathrm{MHz}$. We implemented an interferometric heterodyne detection using a reference beam to detect the absolute value of the FWM amplitude with temporal resolution. The excitation pulses were linearly cross-polarized resulting a linearly polarized PE signal ${ }^{26}$. A detailed description of the experimental technique can be found elsewhere ${ }^{20}$.

In the experiment, the temporal profiles of the PE were measured as a function of the laser pulse amplitude. Figure 1 summarizes the experimental data obtained when the amplitude of the first excitation pulse is varied. This pulse amplitude is proportional to $\sqrt{I_{1}}$, where $I_{1}$ is the time-integrated intensity of the laser pulses. The delay between the centers of rephasing and excitation pulses is set to 66.7 ps. Clearly, footprints of Rabi oscillations are observed in Fig. 1(a). Most strikingly, sizable temporal shifts of the PE maxima for $\pi / 2$ and $3 \pi / 2$ excitation pulses (around $0.8 \mathrm{~mW}^{1 / 2}$ and $1.8 \mathrm{~mW}^{1 / 2}$, respectively) as well as a splitting of the PE profile around $\pi$-pulse amplitude (around $1.3 \mathrm{~mW}^{1 / 2}$ ) become evident in Fig. 1(e), where the corresponding PE transients are plotted. The time-integrated PE signal also manifests Rabi oscillations, as seen from Fig. 1(d), but the oscillations are much less pronounced than in the time-resolved data. Note, that variation of the second pulse amplitude does not lead to any additional modification of the PE temporal profile.

In order to describe the $\mathrm{PE}$ from a $\mathrm{QD}$ ensemble we calculate the temporal evolution of a set of $2 \times 2$ density matrices interacting with two sequent laser pulses and analyze the resulting macroscopic polarization of the medium, $P$, given by the mean value of the dipole moment operator $\hat{d} \rho$ :

$$
P=\operatorname{Tr}(\hat{d} \rho) .
$$

Here, $\rho$ is the density matrix of the TLS and $\operatorname{Tr}$ is the trace of the matrix. The temporal evolution of the density matrix is described by the Lindblad equation: $i \hbar \dot{\rho}=[\hat{H}, \rho]+\Gamma$, where $\hat{H}$ is the Hamiltonian of the 
system and $\Gamma$ describes relaxation processes phenomenologically. We consider the case, when the individual oscillator coherence time $T_{2} \gg \tau_{12}$, which is justified by $T_{2}$ being ultimately limited by the exciton lifetime of about 0.5 ns. Thus, the only consequence of relaxation in the system is the $\mathrm{PE}$ exponential decay $\sim \exp \left(-2 \tau_{12} / T_{2}\right)$. Therefore, we will neglect relaxation considering $\Gamma=0$, for simplicity.

In our model, the Hamiltonian consists of two parts: $\hat{H}=\hat{H}_{0}+\hat{V}$, where $\hat{H}_{0}$ describes the unperturbed system and $\hat{V}=-\hat{d} E$ gives the interaction with light. We assume rectangular shaped pulses, each with with duration $\tau_{p}$. During the optical excitation, we calculate the ensemble dephasing due to the detuning of every individual oscillator with frequency $\omega_{0}$ from the light frequency $\omega: \Delta=\omega-\omega_{0}$. In the rotating-wave approximation, this defines the optical nutation of the TLS on the Bloch sphere with a generalized Rabi frequency $\tilde{\Omega}=\sqrt{\Omega^{2}+\Delta^{2}}$, where $\Omega=2\left|E_{0} d\right| / \hbar$ is proportional to the optical field amplitude $E_{0}$. Accordingly, the analysis of the coherent optical response of the system includes the following steps: first, we calculate the interaction of the unperturbed system with the first excitation pulse $\left(\Omega=\Omega_{1}\right)$ acting during time $\tau_{p}$, then the system evolves freely in absence of excitation $(\Omega=0)$ during the time interval $\tau_{12}$, followed by the excitation with the second rephasing pulse $\left(\Omega=\Omega_{2}\right)$ during time $\tau_{p}$. Finally, we analyze the macroscopic polarization of the QDs that is available for PE pulse formation by integrating the individual responses over the inhomogeneous ensemble of TLS, defined by a Gaussian distribution with standard deviation $\delta$. Within these approximations, it is possible to perform the analysis analytically and calculate the following expression for the modulus of the detected excitationdependent part of the PE amplitude ${ }^{27}$ :

$$
\begin{aligned}
& \left|P_{P E}\left(t^{\prime}\right)\right| \sim \mid \int_{-\infty}^{\infty} \exp \left(-\frac{\Delta^{2}}{2 \delta^{2}}\right) \frac{\Omega_{1} \Omega_{2}^{2}}{\tilde{\Omega}_{1} \tilde{\Omega}_{2}^{2}} \sin ^{2} \frac{\tilde{\Omega}_{2} \tau_{p}}{2} \\
& \times\left[\frac{2 \Delta}{\tilde{\Omega}_{1}} \sin ^{2} \frac{\tilde{\Omega}_{1} \tau_{p}}{2} \sin \Delta t^{\prime}+\sin \tilde{\Omega}_{1} \tau_{p} \cos \Delta t^{\prime}\right] d \Delta \mid
\end{aligned}
$$

Here $t^{\prime}=t-2\left(\tau_{12}+\tau_{p}\right)$, and $\tilde{\Omega}_{1,2}$ are the generalized Rabi frequencies of the first and the second pulse, respectively. In this notation, delay between the exciting pulse centers used in experiment is $\tau_{12}+\tau_{p}$ and expected PE arrival time according to the standard treatment is $t^{\prime}=0$. This equation is similar to the one obtained for spin echoes measured in highly inhomogeneous fields ${ }^{21,22}$. It can be easily seen, that variation of the second, rephasing pulse amplitude and, hence, $\Omega_{2}$ does not affect the symmetry of the PE profile. Therefore, we will consider only the effect coming from variation of the first, excitation pulse amplitude.

Figure 2 shows results of theoretical calculations performed using Eq. (2) for two ensembles with different widths of the inhomogeneity: $\tau_{p} \delta=0.12$ and $1.2^{28}$. For an ensemble broadening much narrower than the pulse
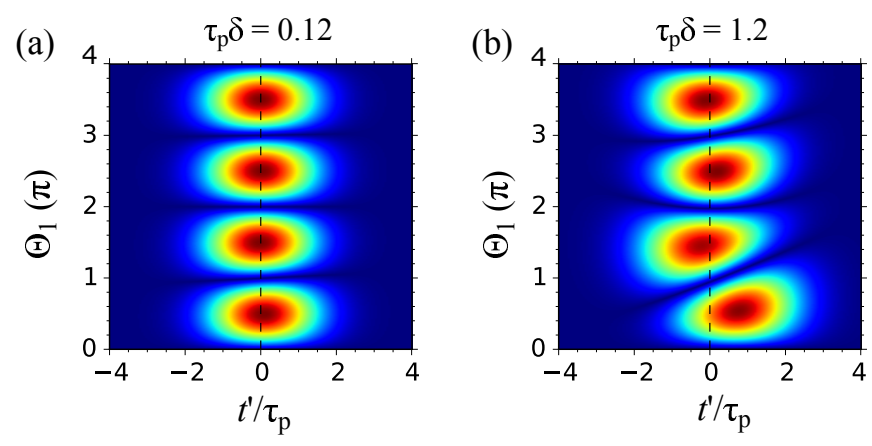

FIG. 2: (Color online) Theoretical calculation of the PE temporal profile as function of the excitation pulse area $\Theta_{1}$ using Eq. (2) for two different ensemble with different inhomogeneity $\delta$ : (a) $\tau_{p} \delta=0.12$; and (b) $\tau_{p} \delta=1.2$. The time scale is normalized by the pulse duration. Dark-blue corresponds to zero signal level, dark-red gives the maximum value.

spectrum, $\tau_{p} \delta=0.12$, clean Rabi oscillations are seen in the PE temporal profile as function of the excitation pulse area $\Theta_{1}=\Omega_{1} \tau_{p}$, shown in Fig. 2(a). In particular the photon echo arrives as expected at twice the separation between rephasing and excitation pulse. This case is equivalent to an ideal Hahn echo, where the echo signal is generated at $2 \tau_{12}$. When, however, the inhomogeneous ensemble width becomes comparable with the pulse spectral width $\left(\tau_{p} \delta=1.2\right)$, strong modifications of the temporal PE profile appear, as can be seen in Fig. 2(b). The key feature of these modifications is the effective temporal shift of the PE amplitude that is particularly prominent for the first two maxima of the Rabi oscillations when increasing the excitation power: for a $\pi / 2$-pulse the $\mathrm{PE}$ maximum is retarded relative to the expected $\mathrm{PE}$ arrival time, while for a $3 \pi / 2$-pulse the PE is advanced. These shifts do not depend on the pulses delay time $\tau_{12}$ in the limit of negligible damping and decrease with further increasing $\Theta_{1}$.

The temporal shifts of the PE can be qualitatively explained using the Bloch sphere representation, as outlined in Fig. 3. In Fig. 3(a), the ideal case of a Hahn echo is given as a reference, where excitation with a negligibly short excitation $\pi / 2$-pulse results in an unshifted echo signal. When the excitation pulse has a finite duration, as shown in Fig. 3(b), oscillators experience dephasing during pulse action, so that the excitation distributes the oscillators along the equator of the Bloch sphere followed by their precession in the equatorial plane. This precession extrapolated to negative times results in focusing all the Bloch vectors on the same meridian, which corresponds to a situation where the maximum macroscopic polarization is shifted to $t<\tau_{p}$. In case of excitation by a $3 \pi / 2$-pulse as shown in Fig. 3(c), after pulse action all oscillators proceed along the equator towards each other, which leads to a shift of the macroscopic polarization maximum to $t>\tau_{p}$. Since the rephasing pulse, which is not necessarily a $\pi$-pulse, acts essentially as a refocusing pulse, which in effect reverses the temporally coherent 

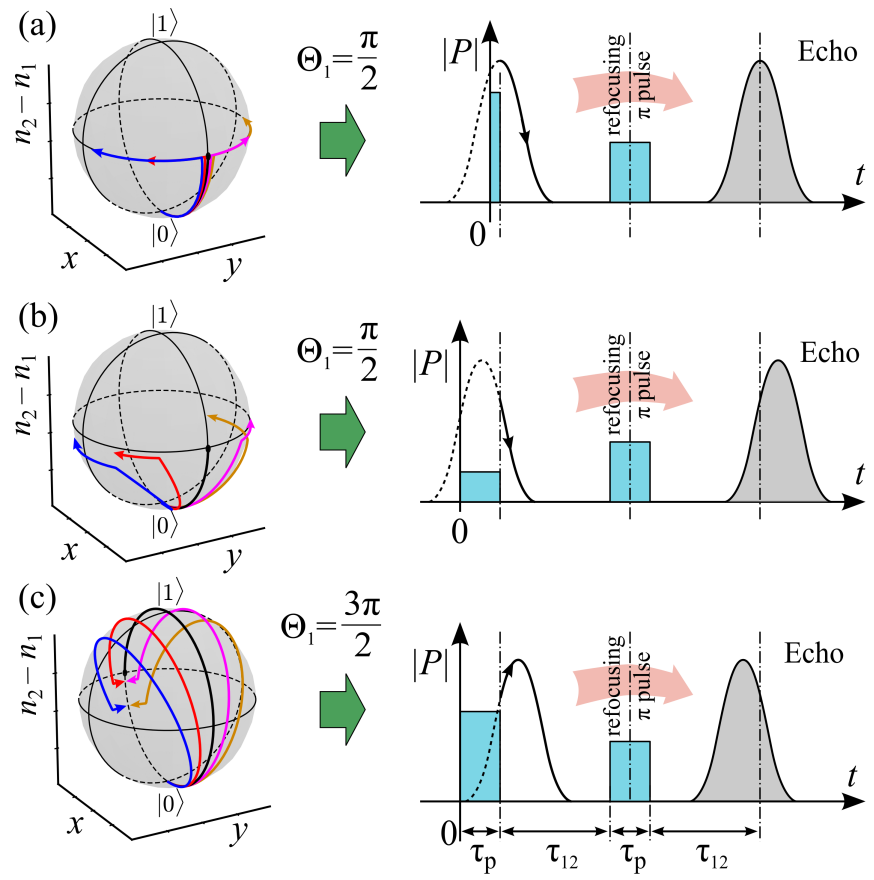

FIG. 3: (Color online) Explanation of the PE timing modifications using the Bloch sphere representation for different excitation pulse configurations: (a) ultrashort $\pi / 2$-pulse: case of Hahn echo. (b) $\pi / 2$-pulse: retarded echo. (c) $3 \pi / 2$-pulse: advanced echo. The colored lines on the Bloch spheres (left panels) denote different trajectories of the Bloch vectors of individual oscillators with different detuning from the laser frequency in directions indicated by the arrows. The right panels show the temporal evolution of the modulus of the macroscopic polarization, $|P|$, after excitation pulse action. The dashed lines denote extrapolation of $|P|$ to $t<\tau_{p}$.

evolution of the ensemble oscillators, the dynamics of the macroscopic dipole moment around the expected echo arrival time, $2\left(\tau_{12}+\tau_{p}\right)$, is similar to a mirrored copy of its dynamics around the time $t=\tau_{p}$, as shown by the right panels of Fig. 3. Thus, the PE pulse is retarded for the a $\pi / 2$-excitation pulse and advanced for a $3 \pi / 2$ pulse, when the ensemble dephasing during pulse action is sufficiently strong. This can be achieved either by high inhomogeneity in the excited ensemble and/or long pulse duration.

The analysis of the experimental data in Fig. 1(a) was performed using the theoretical modeling of the PE effect at varying excitation pulse amplitudes after Eq. (2). The adjustable parameters are $\delta$ and $\tau_{p}$. The theoretical fit depicted in Fig. 1(b) gives a pulse duration $\tau_{p} \approx 2.9 \mathrm{ps}$ and an inhomogeneous ensemble full-width at half-maximum $2 \sqrt{2 \ln 2} \hbar \delta \approx 0.65 \mathrm{meV}$. This broadening is much smaller than the PL linewidth of the QD structure $(6.5 \mathrm{meV})$ indicating that a much narrower subensemble contributes to the PE compared to the ensemble contributing to the PL emission. The origin of this discrepancy will be subject of further studies. A possible reason might be the interaction with the microcavity. The evident Rabi oscillations pattern in the PE sequence tells that QD structures under resonant excitation can be well described by TLS.

In conclusion, the importance of the oscillator detuning from the optical frequency in forming the macroscopic coherent response in semiconductor QDs was addressed in literature already before ${ }^{23,24}$. However, no consequence of the timing of the associated PE had been reported. The essential consequence of our study is that even significantly inhomogeneous semiconductor systems still demonstrate a comprehensible coherent behavior, but great care needs to be exercised to consider the consequences of the inhomogeneity in detail. When doing so, such systems are very well suited candidates for the coherent manipulations in coherent optoelectronics. In particular, the observed temporal shifts of the PE amplitude need to be taken into account when a precise timing of the optical signals matters. Our findings have strong impact on interpretation of two-dimensional Fourier transform images, which are currently widely used because of their intuitive visualization of underlying physics ${ }^{8,25}$. The complex-valued envelope of the PE-transients from strongly inhomogeneous systems results in a changeover of the corresponding 2D image from a resonance profile in case of a Hahn echo to a dispersive profile (see Supplementary materials).

\section{Acknowledgments}

Authors thank Gleb Kozlov for fruitful discussions. We acknowledge the financial support by the Deutsche Forschungsgemeinschaft and the Russian Foundation of Basic Research in the frame of the ICRC TRR 160 (RFBR project No. 15-52-12016 NNIO_a). The project SPANGL4Q acknowledges financial support from the Future and Emerging Technologies (FET) programme within the Seventh Framework Programme for Research of the European Commission, under FET-Open grant no. FP7-284743. S.V.P. thanks the Russian Foundation of Basic Research for partial financial support (contract no. 14-02-31735 mol_a). S.V.P. and I.A.Yu. acknowledge partial financial support from the Russian Ministry of Science and Education (contract no. 11.G34.31.0067) and SPbU (Grant No. 11.38.213.2014). M.B. acknowledges support from the Russian Ministry of Science and Education (contract number 14.Z50.31.0021).
* Electronic address: svp@bk.ru

${ }^{1}$ K. D. Greve, D. Press, P. L. McMahon, and Y. Yamamoto,
Reports on Progress in Physics 76, 092501 (2013).

2 D. Kim, S. G. Carter, A. Greilich, A. S. Bracker, and 
D. Gammon, Nature Physics 7, 223 (2011).

3 E. Biolatti, R. C. Iotti, P. Zanardi, and F. Rossi, Phys. Rev. Lett. 85, 5647 (2000).

4 X. Li, Y. Wu, D. Steel, D. Gammon, T. H. Stievater, D. S. Katzer, D. Park, C. Piermarocchi, and L. J. Sham, Science 301, 809 (2003).

5 M. Kroutvar, Y. Ducommun, D. Heiss, M. Bichler, D. Schuh, G. Abstreiter, and J. J. Finley, Nature 432, 81 (2004).

${ }^{6}$ L. Langer, S. Poltavtsev, I. A. Yugova, M. Salewski, Y. D. R., G. Karczewski, T. Wojtowicz, I. Akimov, and M. Bayer, Nature Photonics 8, 851 (2014).

7 P. Borri, W. Langbein, S. Schneider, U. Woggon, R. L. Sellin, D. Ouyang, and D. Bimberg, Phys. Rev. Lett. 87, 157401 (2001).

${ }^{8}$ G. Moody, R. Singh, H. Li, I. A. Akimov, M. Bayer, D. Reuter, A. D. Wieck, and S. T. Cundiff, Phys. Rev. B 87, 045313 (2013).

9 H. Tahara, Y. Ogawa, F. Minami, K. Akahane, and M. Sasaki, Phys. Rev. B 89, 195306 (2014).

10 Y. Shen, The Principles of Nonlinear Optics (WileyInterscience, New York, 1984).

11 D. S. Chemla and J. Shah, Nature 411, 549 (2001).

12 J. Shah, Ultrafast Spectroscopy of Semiconductors and Semiconductor Nanostructures, vol. 115 of Springer Series in Solid-State Sciences (Springer Science \& Business Media, 1999).

13 G. Noll, U. Siegner, S. G. Shevel, and E. O. Göbel, Phys. Rev. Lett. 64, 792 (1990).

14 A. Zrenner, E. Beham, S. Stufler, F. Findeis, M. Bichler, and G. Abstreiter, Nature 418, 612 (2002).

15 T. H. Stievater, X. Li, D. G. Steel, D. Gammon, D. S, Katzer, D. Park, C. Piermarocchi, and L. J. Sham, Phys. Rev. Lett. 87, 133603 (2001).
16 A. J. Ramsay, T. M. Godden, S. J. Boyle, E. M. Gauger, A. Nazir, B. W. Lovett, A. M. Fox, and M. S. Skolnick, Phys. Rev. Lett. 105, 177402 (2010).

17 A. Gamouras, R. Mathew, S. Freisem, D. G. Deppe, and K. C. Hall, Nano Letters 13, 4666 (2013).

18 N. Allen and J. H. Eberly, Optical Resonance and TwoLevel Atoms, chapter 9 (New York, Wiley, 1975).

19 V. V. Samartsev, R. G. Usmanov, G. M. Ershov, and B. S. Khamidullin, Sov. Phys. JETP 47, 1030 (1978).

20 L. Langer, S. V. Poltavtsev, I. A. Yugova, D. R. Yakovlev, G. Karczewski, T. Wojtowicz, J. Kossut, I. A. Akimov, and M. Bayer, Phys. Rev. Lett. 109, 157403 (2012).

21 A. L. Bloom, Phys. Rev. 98, 1105 (1955).

22 I. G. Kiliptari and V. I. Tsifrinovich, Phys. Rev. B 57, 11554 (1998).

23 B. Patton, U. Woggon, and W. Langbein, Phys. Rev. Lett. 95, 266401 (2005).

24 A. J. Ramsay, R. S. Kolodka, F. Bello, P. W. Fry, W. K. Ng, A. Tahraoui, H. Y. Liu, M. Hopkinson, D. M. Whittaker, A. M. Fox, et al., Phys. Rev. B 75, 113302 (2007).

25 S. Cundiff, T. Zhang, A. Bristow, D. Karaiskaj, and D. Xinccan, Accounts of Chemical Research 42, 1423 (2009).

26 Accurate theoretical description requires use of a $4 \times 4$ density matrix formalism in order to account for the linear polarizations as shown in Ref. ${ }^{20}$. It was checked, however, that the effect of the PE shifts demonstrated here is valid for any polarization configuration.

27 See Supplemental Material containing details of the theoretical approach.

28 In the calculations, dimensionless units are used for convenience for the frequency and the time scales. In these units, the pulse spectral width is equal to 1 . 